Revista peruana de biología 26(4): 481 - 490 (2019) doi: http://dx.doi.org/10.15381/rpb.v26i4.15990 ISSN-L 1561-0837; eISSN: 1727-9933

Universidad Nacional Mayor de San Marcos

\section{Variación de la abundancia de Lama guanicoe en el Parque Nacional Bosque Fray Jorge, Coquimbo, Chile y su relación con la cobertura vegetal}

\author{
Variation of the abundance of Lama guanicoe in Bosque \\ Fray Jorge National Park Coquimbo, Chile, and its relation \\ with plant cover
}

\section{TRABAJOS ORIGINALES}

$\begin{array}{ll}\text { Presentado: } & 16 / 04 / 2019 \\ \text { Aceptado: } & 25 / 10 / 2019 \\ \text { Publicado online: } & 16 / 12 / 2019\end{array}$

Correspondencia:

*Autor para correspondencia

Víctor Cook-Mena: victor.cook@conaf.cl César Chávez-Villavicencio: cchavez@ucn.cl Paula Martínez-Palma: paula.martinez@conaf.cl ElierTabilo-Valdivieso: etabilo@centroneotropical.org

Otros datos de los autores / biografía:

ORCID CC-V: 0000-0003-2185-8308

ORCID ET-V: 0000-0002-8217-7867

1 Parque Nacional Bosque Fray Jorge, Corporación Nacional Forestal Región de Coquimbo, Regimiento Arica 901, Coquimbo, Chile.

2 Centro Neotropical de Entrenamiento en Humedales, Carmen Quiroga 421, La Serena. Coquimbo - Chile.

3 Programa de Doctorado en Biología y Ecología Aplicada, Universidad Católica del Norte, Universidad de La Serena. Campus Guayacán, Larrondo 1281, Coquimbo - Chile

Citación:

Cook-Mena V., C. Chávez-Villavicencio, P. MartínezPalma, E. Tabilo-Valdivieso. 2019. Variation of the abundance of Lama guanicoe in Bosque Fray Jorge National Park (Coquimbo - Chile) and its relation with plant cover. Revista peruana de biología 26(4): 481 - 490 (Diciembre 2019). doi: http://dx.doi. org/10.15381/rpb.v26i4.15990

Palabras clave: Áreas protegidas; Camélidos andinos; Grandes herbívoros; Restauración de poblaciones: Reintroducción animal; Translocación de fauna. Keywords: Andean camelids; Animal reintroduction; Fauna translocation; Large herbivore; Population restoration.

\section{Víctor Cook-Mena ${ }^{1}$, César Chávez-Villavicencio*2,3, Paula Martí- nez-Palma ${ }^{1}$, Elier Tabilo-Valdivieso ${ }^{2,3}$}

1 Parque Nacional Bosque Fray Jorge, Corporación Nacional Forestal Región de Coquimbo, Chile.

2 Centro Neotropical de Entrenamiento en Humedales, La Serena, Chile.

3 Programa de Doctorado en Biología y Ecología Aplicada, Universidad Católica del Norte, Universidad de La Serena, Chile.

\section{Resumen}

Lama guanicoe se distribuyó en los Andes de Chile desde Arica y Parinacota hasta Magallanes. Actualmente su distribución está fragmentada con poblaciones en las regiones de Tarapacá, Coquimbo, Aysén y Magallanes. En el Parque Nacional Bosque Fray Jorge (Coquimbo), las últimas poblaciones de guanacos silvestres se observaron el año 1920. En 1994 se liberaron ocho individuos en el Parque de los cuales cinco sobrevivieron. Desde entonces se realizaron conteos ocasionales de la población reintroducida. En noviembre de 2013 se elaboró una metodología de monitoreo con base en transectos de conteo. Los objetivos fueron analizar los cambios históricos de la abundancia poblacional de guanacos en el Parque y determinar el uso y selección de hábitat. Se emplearon modelos lineales para analizar las tendencias poblacionales y el Cociente de Selección de Manly para la selección de hábitat. Se evidenció incremento de la población hasta 33 individuos. El principal uso que dieron a los tipos de cobertura fue la alimentación y seleccionaron el Matorral Desértico Mediterráneo Interior de Heliotropium stenophyllum y Fluorensia thurifera. Se recomienda marcar los guanacos con collares satelitales para hacer un seguimiento de los movimientos de las tropas, establecer territorios y medir el tamaño poblacional.

\section{Abstract}

Lama guanicoe was distributed in the Andes of Chile from Arica and Parinacota to Magallanes. Currently its distribution is fragmented with populations in Tarapaca, Coquimbo, Aysen and Magallanes Regions. In the Bosque Fray Jorge National Park (Coquimbo), the last populations of wild guanacos were observed in the year 1920 In 1994, eight individuals were released in the Park, of which five survived. Since then, occasional counts of the reintroduced population were made. In November 2013, a monitoring methodology was developed based on count transects. The objectives were to analyze the historical changes in the population of guanacos in the Park and determine the use and selection of habitats. Linear models were used to analyze population trends and the Manly Selection Ratio for habitat selection. There was an increase in the population to 33 individuals. The main use that they gave to the types of cover was the feeding and they selected the Mediterranean Mediterranean Desert Scrub of Heliotropium stenophyllum and Fluorensia thurifera. It is recommended to mark the guanacos with satellite collars to monitor the movements of the troops, establish territories and measure the population size. 


\section{Introducción}

En Chile, antes de la llegada de los españoles, Lama guanicoe (guanaco) se distribuía a lo largo de los Andes, desde la Región de Arica y Parinacota en el norte hasta la Región de Magallanes en el sur (Grimberg 2010). En la actualidad, su distribución está fragmentada con poblaciones estables en las regiones de Tarapacá, Aysén y Magallanes (Marín 2008). Sin embargo, en la Región de Coquimbo también hay poblaciones que se encuentran en las cordilleras de Illapel y San Agustín en las localidades de Cuncumén y Chillepín (Grimberg 2010), mientras que en el Parque Nacional Bosque Fray Jorge (PNBFJ), los pobladores locales refieren que las últimas poblaciones de guanacos silvestres se observaron alrededor del año 1920 y desde entonces no se volvió a observar ni en el Parque ni en los alrededores (Schamberger \& Fulk 1974, Cook 2012).

Con el antecedente de la presencia de la especie en el Parque, en noviembre de 1994, un particular donó tres individuos adultos (un macho y dos hembras) al PNBFJ, que fueron liberados en la Quebrada La Escondida, un sector del Parque que presentaba condiciones de hábitat adecuado y vigilancia permanente de los Guardaparques (Cook 2012). La excesiva agresividad del macho que mató a una de las hembras, lo llevó a ser sacrificado por personal del Parque (Cook 2012). En diciembre de 1994, el Servicio Agrícola Ganadero de la Región de Valparaíso (Chile), liberó en la quebrada Las Vacas, otro grupo de cinco individuos adultos (dos machos y tres hembras), de los cuales un macho también fue sacrificado por su agresividad, quedando finalmente dentro del PNBFJ un grupo de cinco individuos (un macho y cuatro hembras) (Cook 2012).

La liberación de estos individuos dentro del PNBFJ, sin ser marcados para identificarlos en un posterior seguimiento o monitoreo, como Barri y Cufré (2014) sugieren que se debe hacer, no permitió realizar un control efectivo de las actividades y el éxito de la medida. La reintroducción es una forma de manejo ecológico cada vez más frecuente en el mundo (Beck et al. 1994, IUCN 2013), donde el monitoreo constituye una parte relevante en la restauración de una especie, permitiendo inferir ajustes en su manejo y rol ecológico en los sistemas naturales (Estes 1996, Seddon 1998). Los aspectos a considerar en el monitoreo para el manejo, conservación y estado poblacional son los patrones de uso de hábitat y obtención del alimento, reproducción y defensa contra depredadores (Herrera 1999). A pesar de no haber marcado los individuos liberados, en diciembre de 1994 los Guardaparques iniciaron el registro de avistamientos de los individuos de la especie dentro del PNBFJ durante los patrullajes rutinarios dentro de los senderos establecidos, que no cubrían la totalidad del Parque, además de no seguir un diseño de muestreo estadístico apropiado (Cook 2012).

Debido a que la liberación de los primeros individuos fue un intento por recuperar la especie dentro de un área protegida en Chile y los aspectos a considerar descritos por Herrera (1999) en el párrafo anterior, los objetivos de este trabajo fueron analizar los cambios en la abundancia poblacional de guanacos en el PNBFJ desde el año 1994 hasta el año 2017, y determinar el uso y selección de la cobertura vegetal que compone su hábitat dentro del Parque.

\section{Material y métodos}

Área de estudio.- El Parque Nacional Bosque Fray Jorge $\left(30^{\circ} 38^{\prime} \mathrm{S}-71^{\circ} 40^{\prime} \mathrm{W}\right)$ se ubica en la comuna de Ovalle, Provincia de Limarí, Región de Coquimbo (Fig. 1), a $110 \mathrm{~km}$ al sur de la comuna de La Serena, comprende a la zona mediterránea árida con influencias oceánicas y una extensión de 9.959 ha (Squeo et al. 2001). En el área se reconocen tres subunidades geomorfológicas que corresponden a las terrazas litorales, el cordón de Altos de Talinay y el sector oriental del mismo (Paskoff 1993, Pouget et al. 1996).

La estrecha extensión de las Terrazas Marinas de Abrasión (aproximadamente $5 \mathrm{~km}$ ) es interrumpida abruptamente por los Macizos de la Cordillera de la Costa (Paskoff 1993), específicamente por los Altos de Talinay que alcanzan cerca de $700 \mathrm{~m}$ de altitud (Novoa \& López 2001). En áreas donde el relieve supera los 400 $\mathrm{m}$ de altitud, las masas de aire marino son interceptadas y forzadas a ascender por la vertiente occidental, con lo cual disminuye su capacidad de retención de humedad, creándose un ambiente lo suficientemente húmedo como para sostener a las comunidades vegetales presentes (Rundel \& Mahu 1976, Marquet et al. 1998). Según la clasificación de pisos vegetacionales de Luebert \& Pliscoff (2009), el PNBFJ presenta tres tipos de cobertura vegetal: Matorral Desértico Mediterráneo Interior de $\mathrm{He}$ liotropium stenophyllum y Fluorensia thurifera (3274.6 ha), Matorral Desértico Mediterráneo Costero de Bahia ambrosioides y Puya chilensis (2333.2 ha) y Matorral Desértico Mediterráneo Costero de Oxalis gigantea y Heliotropium stenophyllum (3337.4 ha).

Cambios poblacionales.- Se realizó una revisión de la base de datos de conteos de abundancia de guanacos elaborada por los Guardaparques del PNBFJ en sus recorridos de rutina, para conocer, a pesar de ser registros ocasionales, la cantidad de individuos de la especie desde su liberación en noviembre de 1994 hasta antes de dar inicio a un programa formal de monitoreo en el año 2013. Este programa formal surgió producto de un taller realizado en el PNBFJ con el equipo de Guardaparques en septiembre de 2013, en este taller, se diseñó una metodología que estableció que la manera de conducir el conteo de guanacos dentro del Parque era recorrer, el primer día, transectos en los sitios de avistamiento regular de la especie y, en un segundo día, los sitios donde no se tenía referencias de avistamiento (Cook et al. 2013, Cook 2016) (Fig. 1). A partir de noviembre de 2013, con el diseño establecido y probado, se realizó un registro de datos por año cada mes de octubre hasta el año 2017.

Los transectos establecidos para el monitoreo se ubicaron de acuerdo a criterios de accesibilidad, personal disponible, costos operativos y paso por los tres pisos vegetacionales presentes en el Parque. Los transectos tu- 


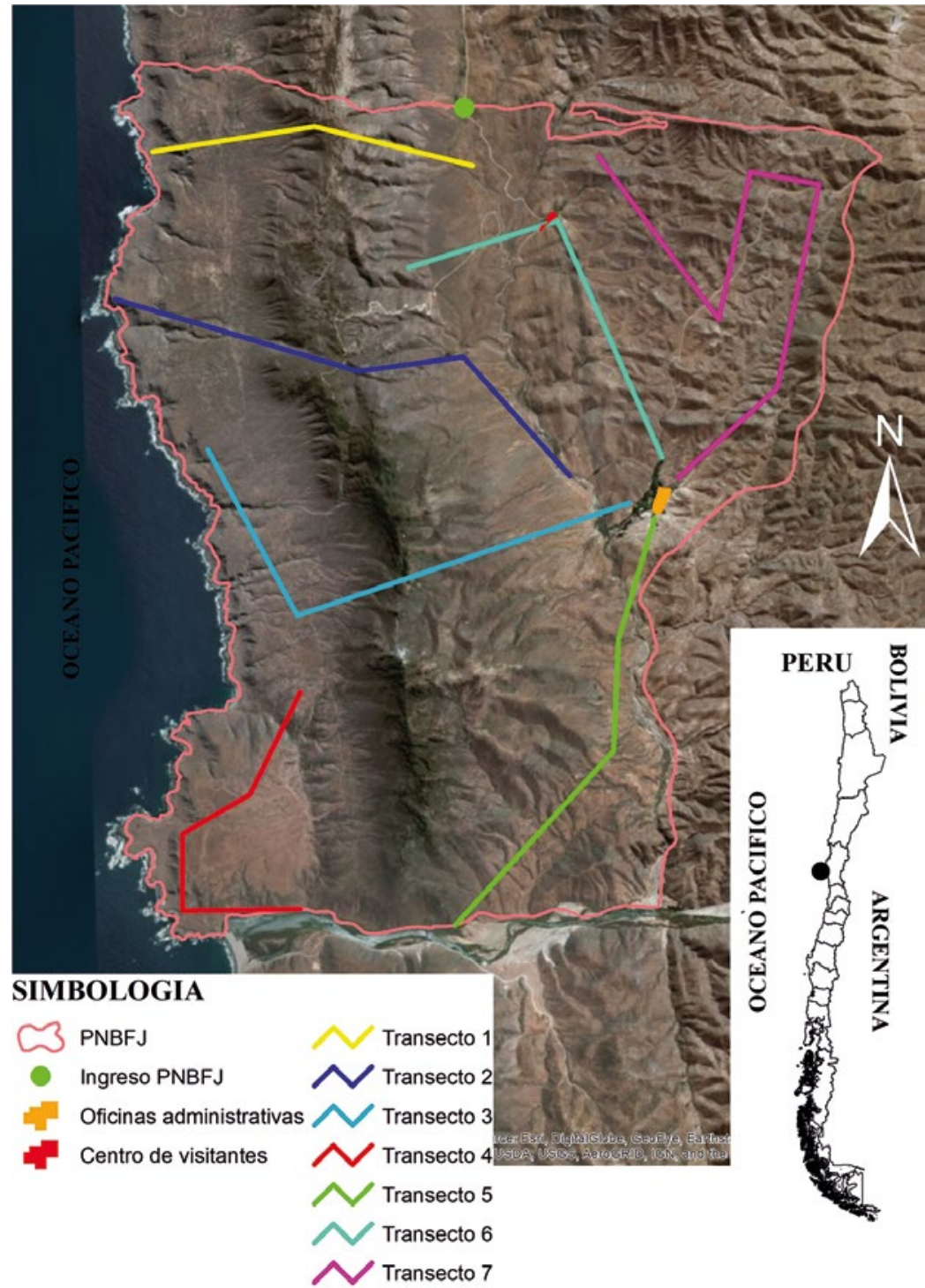

Figura 1. Ubicación del Parque Nacional Bosque Fray Jorge (PNBFJ) y disposición de los transectos de conteo de Lama guanicoe.

vieron ancho variable (hasta $5 \mathrm{~km}$ ) y la detección se realizó con el uso de binoculares sumado a la experiencia de los Guardaparques. El primer día se recorrieron los transectos uno $(4.5 \mathrm{~km})$, dos $(5.3 \mathrm{~km})$, tres $(5.7 \mathrm{~km})$ y cuatro $(6.5 \mathrm{~km})$. El segundo día se recorrieron los transectos cinco $(7.2 \mathrm{~km})$, seis $(7.5 \mathrm{~km})$ y siete $(10.6 \mathrm{~km})$. Todos los transectos se recorrieron en dirección este - oeste. Cada equipo de conteo estuvo conformado por tres a cuatro personas que llevaban un GPS, un radio para comunicarse con otros equipos para evitar dobles conteos y el apoyo del personal contra incendios forestales ubicados en la parte alta del cerro Mozambique $\left(30^{\circ} 39^{\prime} \mathrm{S}-7^{\circ} 36^{\prime}\right.$ 0) a $540 \mathrm{msnm}$. Los transectos fueron recorridos a pie con excepción de los transectos 2 y 6 que se recorrieron a caballo debido a la dificultad que presentaron para el desplazamiento.

Los datos obtenidos con la metodología de monitoreo implementada en septiembre de 2013 sumados al dato de la población inicial liberada en 1994 se analizaron con una regresión de Poisson para establecer si hubo incremento significativo del número de individuos. También se determinó la diferencia entre el promedio de machos y hembras con una prueba de t-student de Welch (diferencia de promedios entre grupos con varianzas diferentes), para conocer la relación entre los sexos dentro del Parque. Todos los cálculos se realizaron con el paquete Rcmdr del programa R (R Development Core Team 2018).

Uso y selección de cobertura vegetal.- El uso de la cobertura vegetal se determinó con la sumatoria de las ubicaciones de los guanacos, revolcaderos, letrinas o huellas de acuerdo al Diseño de Tipo I de Manly (Manly et al. 2003). La cobertura vegetal, para este estudio, correspondió con los tres tipos descritos para el Parque por Luebert \& Pliscoff (2009). La selección de la cobertura vegetal se determinó con base en la desproporción entre el uso que correspondió a los datos acumulados registrados entre 2013 y 2017 y la disponibilidad de la cobertura vegetal (Manly et al. 2003). La selección se calculó con el Cociente de Selección de Manly (Wi) usando el paquete Adehabitat del programa R (R Development Core Team 2018). El cociente de selección de Manly in- 
dica selección a favor a valores mayores que uno, valores menores que uno indican selección en contra y uno indica no selección, en ningún caso significa no uso (Manly et al. 2003). Esta función evalúa la selección del componente con la prueba de uso aleatorio de recursos según el estadístico de Chi-cuadrado de Pearson, en el Diseño de Tipo I los resultados del Chi-Cuadrado del cociente de selección, prueban la hipótesis de que las proporciones de selección son en promedio iguales a cero (Manly et al. 2003). La precisión de los estimados se verificó con el intervalo de confianza de Bonferroni al 95\% por ser más riguroso que el valor de p (Johnson 1999, Sarria \& Silva 2004). Además de los datos de ubicación, se consideró la actividad que realizaban los individuos (alimentación, desplazamiento, reposo o vigilancia por parte del macho alfa), los componentes de la dieta se determinaron por observación directa del consumo.

\section{Resultados}

Cambios poblacionales.- La base de datos de los Guardaparques del PNBFJ, entre los años 1994 y 2012, registró variación en la población de guanacos posterior a la liberación (Fig. 2). Sin embargo, el registro de 1995 da cuenta de la duplicación de la abundancia de los guanacos en el PNBFJ lo que podría deberse a un doble conteo al no seguir una metodología adecuada, por lo que no es posible establecer incremento o disminución en ese período de evaluación debido a que esta situación vuelve desconfiable a los datos. Lo que sí se pudo establecer con seguridad, es que los animales liberados en el Parque lograron reproducirse dado que se registró la presencia de un juvenil en el año 1995 y desde entonces hasta el año 2012, se fue registrando nuevos individuos juveniles (Fig. 2).

A partir de noviembre de 2013, después de la elaboración de una metodología de muestreo adecuada, se verificó una variación en la población de guanacos, partiendo con la población inicial liberada dentro del Parque en 1994 (Fig. 3), de cinco individuos liberados en el PNBFJ a 33 individuos en octubre de 2016 y 23 individuos en octubre de 2017.
Los análisis estadísticos y sus intervalos de confianza al 95\% mostraron que el número de tropas, individuos, hembras y machos de la población de guanacos del PNBFJ, se incrementó en los últimos 23 años. El número de juveniles en el PNBFJ no mostró evidencia estadística de un incremento en el número de individuos (Tabla 1).

Proporción de sexos.- Normalmente el número de hembras casi siempre fue mayor al número de machos, aunque la variación fue bastante irregular. Sin embargo, la proporción esperada cambió ligeramente en 2014 y 2016 cuando el número de machos superó el conteo del número de hembras, regresando a lo esperado en 2017 (Fig. 4).

Sin embargo, el análisis estadístico no mostró evidencia de una diferencia significativa entre el promedio del número de machos y hembras. El promedio de machos fue de 4.0 individuos, el de hembras fue de 5.0 individuos y la diferencia fue de 1.0 (IC 95\% - $2.0-5.0$ ). La proporción de machos y hembras en promedio fue de 1 macho por cada 1.4 hembras.

Uso y selección de cobertura vegetal.- El principal uso que dieron los guanacos a la cobertura vegetal del PNBFJ fue la alimentación (Fig. 5). De acuerdo a las observaciones directas del equipo de trabajo, el guanaco se alimentó de especies herbáceas, arbustivas, incluso fru-

Tabla 1. Valores de las pendientes de las regresiones de Poisson con respecto a las variables de la población de guanacos del PNBFJ. IC (95\%): Intervalo de confianza al 95\%.

\begin{tabular}{lcc}
\hline & Pendiente & IC (95\%) \\
\hline $\mathrm{N}^{\circ}$ Tropas & 0.26 & $0.11-0.41$ \\
$\mathrm{~N}^{\circ}$ Individuos & 0.03 & $0.02-0.05$ \\
$\mathrm{~N}^{\circ}$ Hembras & 0.08 & $0.03-0.13$ \\
$\mathrm{~N}^{\circ}$ Machos & 0.08 & $0.03-0.12$ \\
$\mathrm{~N}^{\circ}$ Juveniles & 0.08 & $-0.21-0.35$ \\
\hline
\end{tabular}

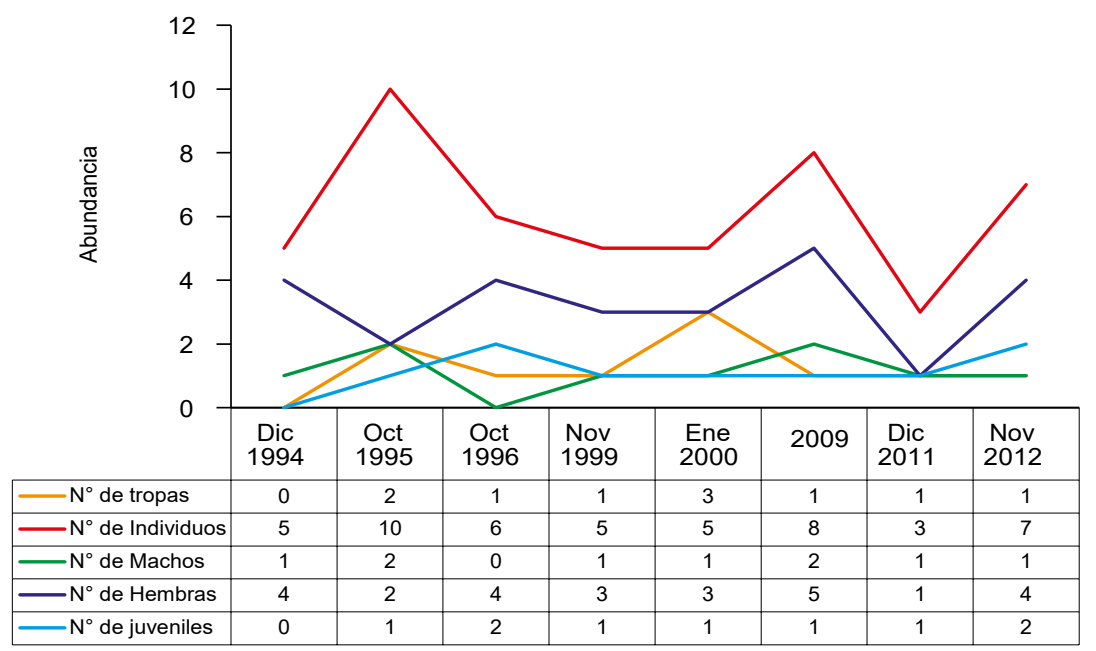

Figura 2. Cambios en la población del guanaco en el PNBFJ según la base de datos de los Guardaparques. 


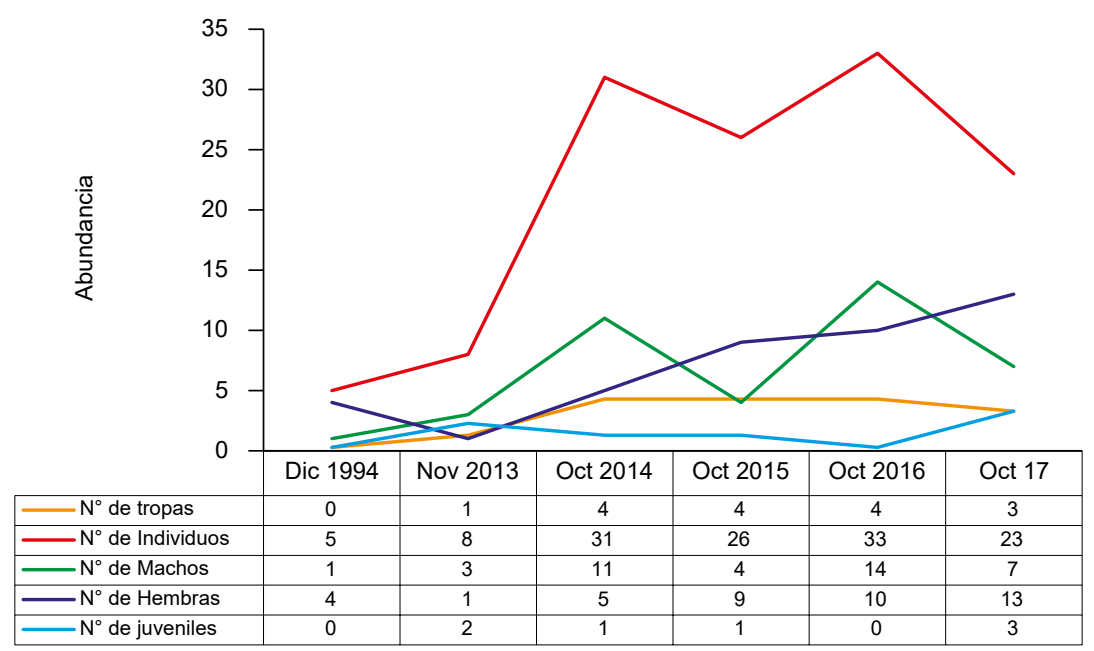

Figura 3. Variación de la población de guanacos en el PNBFJ desde la liberación de cinco individuos en el año 1994 hasta el año 2017.

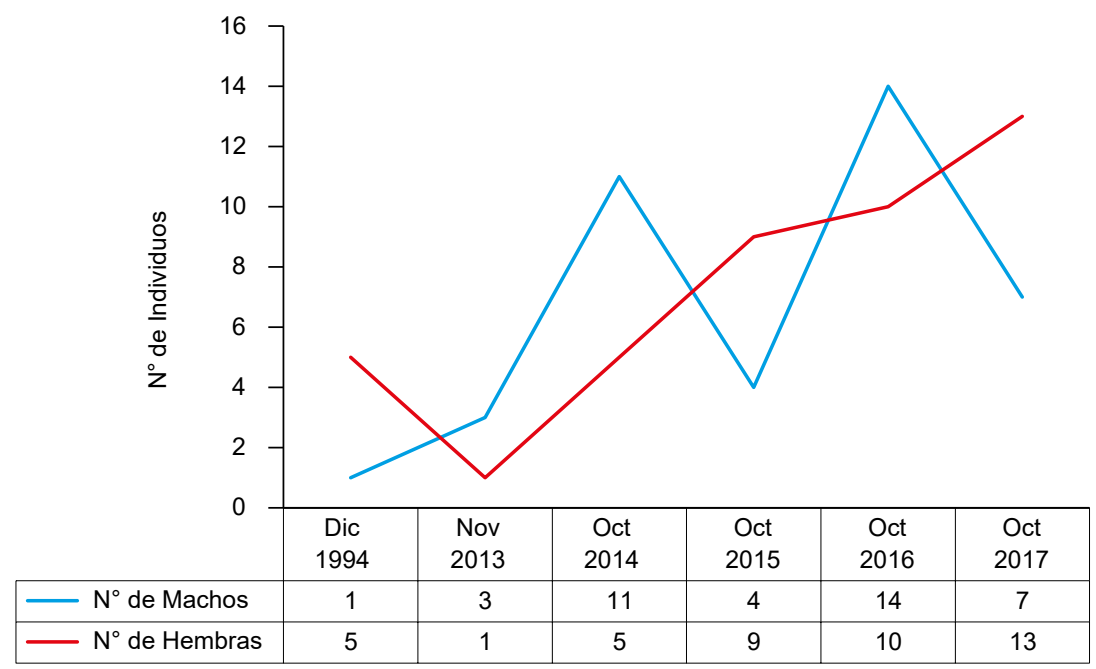

Figura 4. Variación en el tiempo del número de machos y hembras de guanacos del PNBFJ.

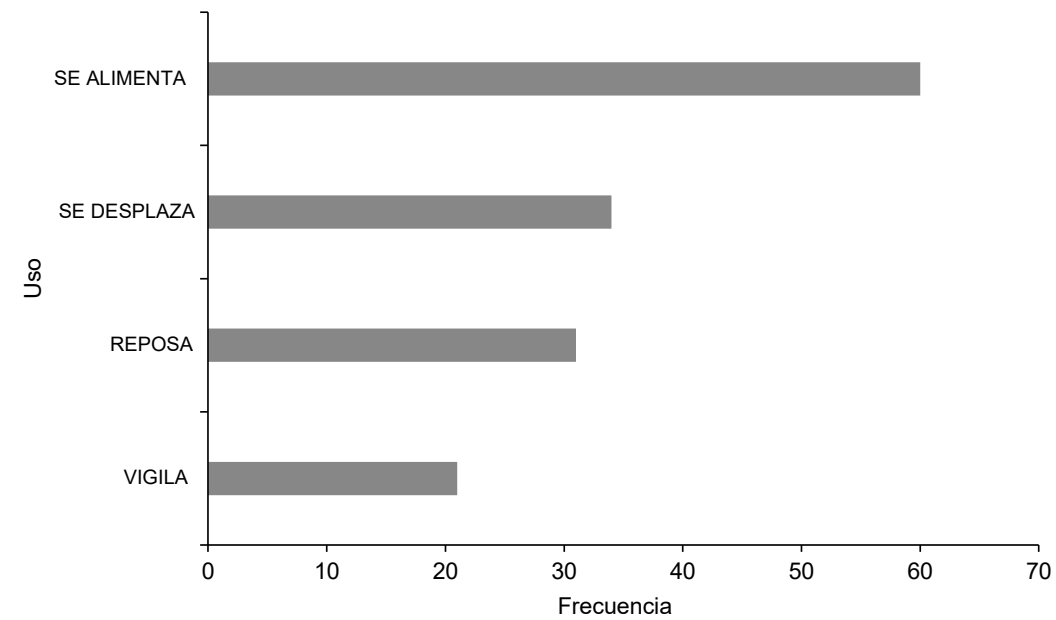

Figura 5. Frecuencia de los usos realizados por los guanacos en el PNBFJ. 
tos de cactáceas. Las especies que fueron parte de la dieta correspondieron a Adesmia sp., Bridgesia sp., Calandrinia sp., Fluorensia sp., Puya sp., Stipa sp., Festuca sp., y Bahia sp., principalmente. Los otros usos con menor frecuencia fueron desplazamientos dentro del Parque, reposo posterior a la alimentación y la vigilancia de la tropa por parte del macho alfa (Fig. 5).

Los guanacos del PNBFJ seleccionaron la cobertura vegetal de Matorral Desértico Mediterráneo Interior de Heliotropium stenophyllum y Fluorensia thurifera que coincide con la subunidad geomorfológica Altos de Talinay. La probabilidad de selección ( $\mathrm{Bi}=0.50$, Tabla 2$)$ de este tipo de cobertura fue prácticamente el doble que la probabilidad de selección de los otros tipos de cobertura. Los tipos de cobertura de Matorral Desértico Mediterráneo Costero de Bahia ambrosioides y Puya chilensis y Matorral Desértico Mediterráneo Costero de Oxalis gigantea y Heliotropium stenophyllum, fueron seleccionados en contra, es decir, los usaron pero no fueron importantes para los guanacos del Parque (Tabla 2. Figs. 6 y 7).

\section{Discusión}

Cambios poblacionales.- Reintroducir especies silvestres en áreas naturales de las cuales desaparecieron es una herramienta muy usada en la biología de la conservación (Soorae 2011). Sin embargo, es necesario que los individuos liberados pasen por un periodo previo de pre-adaptación antes de ser liberado para reducir el estrés de los animales (Barri \& Cufré 2014), si este planteamiento se hubiera conocido y tomado en cuenta en 1994, cuando los guanacos fueron liberados en el PNBFJ, probablemente no habría sido necesario sacrificar a los individuos machos agresivos y se habría evitado la pérdida de estos animales. La experiencia en proyectos de reintroducción indica que es fundamental que se realicen bajo el marco de un monitoreo continuo y manejo adaptativo (Seddon \& Soorae 1999, Armstrong 2007). Sin embargo, realizar el monitoreo de animales reintroducidos involucra marcarlos de alguna manera para poder hacer el seguimiento como se hizo en el Parque Nacional Quebrada Condorito (PNQC) en Argentina (Barri \& Cufré 2014). Los guanacos reintroducidos en el PNBFJ nunca fueron marcados y el seguimiento posterior se hizo siguiendo la certeza y conocimiento de los Guardaparques quienes estaban familiarizados con el grupo liberado. Esta experiencia de reintroducción estimula a emplear a futuro, los estándares de reintroducción que en la actualidad se conocen (periodo previo de pre-adaptación y marcaje básicamente), como se hizo en el PNQC (Barri \& Cufré 2014, IUCN 2013).

Con respecto del cambio en el tamaño de la población de guanacos dentro del PNBFJ, una vez establecido el diseño y la metodología para la toma de datos en septiembre de 2013, los análisis estadísticos mostraron
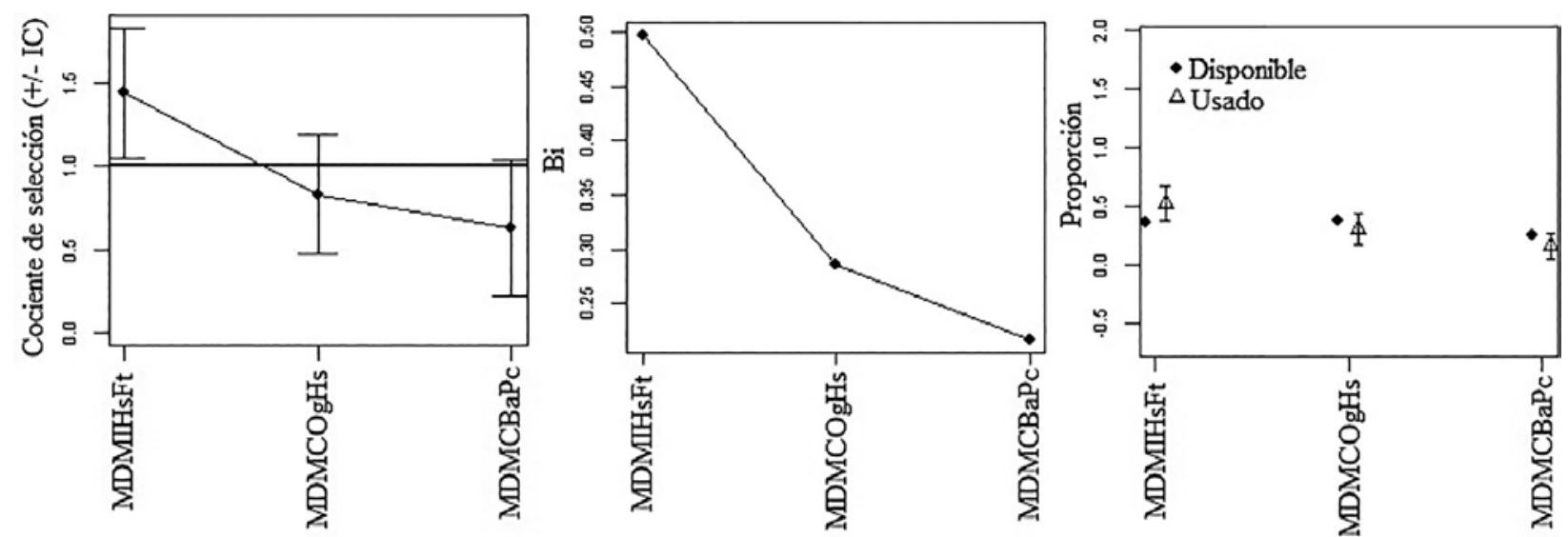

Figura 6. Valores del cociente de selección de Manly. Las barras corresponden a intervalos de confianza de Bonferroni al 95\%. Las iniciales de los tipos de cobertura vegetal provienen de la tabla 2.

Tabla 2. Valores del Cociente de Selección de Manly (“Wi”) para los tipos de cobertura vegetal del PNBFJ. EE: Error Estándar.

\begin{tabular}{|c|c|c|c|c|c|}
\hline Tipo de cobertura vegetal & \% Disponibilidad & Uso & $\begin{array}{l}\text { Cociente de selección "Wi" } \\
\text { (EE) }\end{array}$ & $p$ & $\begin{array}{l}\text { Índice estandarizado } \\
\text { (Bi) }\end{array}$ \\
\hline $\begin{array}{l}\text { Matorral Desértico Mediterráneo Interior de Heliotropium } \\
\text { stenophyllum y Fluorensia thurifera (MDMIHsFt) }\end{array}$ & 36.6 & 29 & $1.44(0.18)$ & 0.02 & 0,5 \\
\hline $\begin{array}{l}\text { Matorral Desértico Mediterráneo Costero de Bahia ambro- } \\
\text { sioides y Puya chilensis (MDMCBaPc) }\end{array}$ & 26.1 & 9 & $0.63(0.19)$ & 0.05 & 0,22 \\
\hline $\begin{array}{l}\text { Matorral Desértico Mediterráneo Costero de Oxalis gigan- } \\
\text { tea y Heliotropium stenophyllum (MDMCOgHs) }\end{array}$ & 37.3 & 17 & $0.83(0.17)$ & 0.31 & 0,29 \\
\hline
\end{tabular}




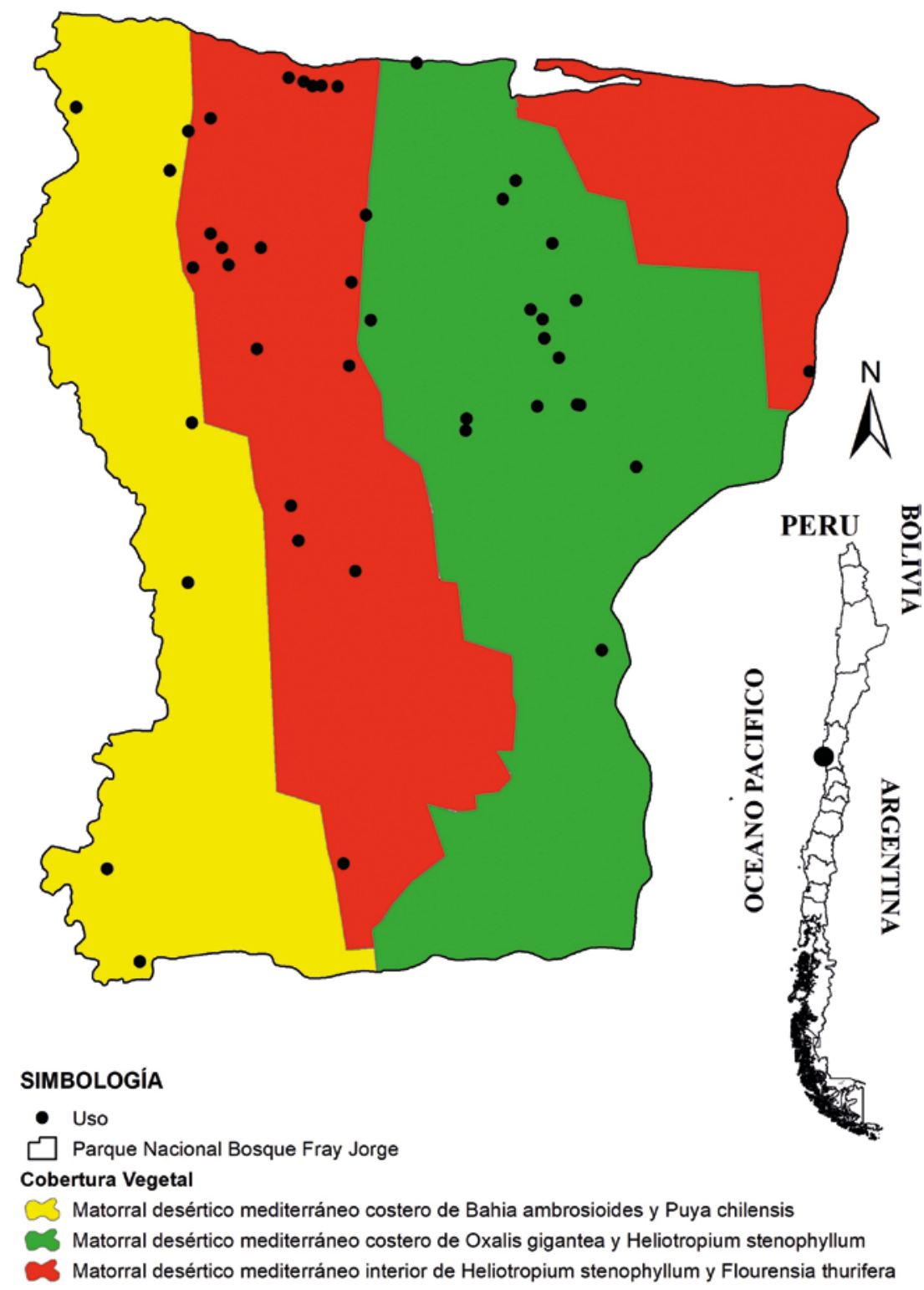

Figura 7. Relación de los puntos de ubicación de usos y cobertura vegetal en el PNBFJ.

que hubo un crecimiento efectivo de la población. Este incremento puede explicarse por la adaptación de los guanacos al Parque debido a la presencia de alimento adecuado, el guanaco como herbívoro generalista de selectividad intermedia, es capaz de consumir la mayoría de las especies de plantas disponibles, tanto pastos como especies leñosas y arbustos (Puig et al. 2001, Baldi et al. 2004). De acuerdo a las observaciones del equipo de trabajo, los guanacos que habitan el PNBFJ se alimentaron de especies herbáceas, arbustivas, incluso frutos de cactáceas. La presencia de alimento y el hábitat adecuado, favorecieron la supervivencia del guanaco y, los nacimientos y muertes, jugaron su rol dentro del grupo reintroducido, junto a otros factores que coinciden con los propuestos por Baldi et al. (2006), los cuales fueron la baja competencia por el alimento con otros herbívoros como vacunos, equinos y caprinos, el buen estado del hábitat, la ausencia de depredadores y la casi nula cacería furtiva en el PNBFJ.
El incremento de la población de guanacos dentro del PNBFJ también implicó un incremento en el número de tropas como ocurrió en el PNQC (Barri \& Fernández 2011). El incremento del número de tropas dentro del PNBFJ en algún momento trajo consigo la necesidad de establecer nuevos territorios, considerando que el guanco es una especie de territorialidad estricta y permanente (Raedeke 1979), lo que llevó a las nuevas tropas a salir de los límites del Parque, dificultando la detección durante los monitoreos, sobre todo en noviembre de 2013 donde solo se registró una tropa. Este dato contrasta fuertemente con los registrados entre noviembre de 2014 y octubre de 2017 cuando se registraron mayor número de tropas. El incremento de los juveniles, también se debe a procesos reproductivos en el PNBFJ. Los juveniles normalmente permanecen hasta los 15 meses de edad en su tropa (Fundación DeSdelChaco 2004) y después de ese tiempo son expulsados. Este conocimiento permite inferir que la tasa reproductiva del guanaco dentro del Parque estaría favoreciendo el recambio de 
los juveniles que fueron expulsados de sus tropas. Considérese que antes de que se produzca el tope de partos, la mayoría de las crías nacidas el año anterior son expulsadas de los grupos familiares por el macho adulto y pasan a formar parte de otros grupos familiares o grupos de machos (Raedeke 1979, Franklin 1983, Puig \& Videla 1995, Sarno et al. 2003).

La proporción de sexos fue diferente a lo esperado, considerando que Cajal (1983) observó una proporción de 2.5:1 a favor de las hembras, similar a la observada por Gader (1982). En cautiverio un macho puede tener entre dos y nueve hembras dentro de su harén (Sarasqueta 2001). Sin embargo, hubo casos en los que la proporción de machos fue superior como en Tierra del Fuego (Argentina) donde la proporción fue de 1.6:1 a favor de los machos (Bonino \& Fernández 1994) similar a la registrada por Raedeke (1978) que fue 1.3 machos por cada hembra. La presencia de machos viejos solitarios (probablemente expulsados de sus tropas) y machos jóvenes solitarios buscando formar su harén coincide con los resultados de Puig \& Videla (1995), relacionado al abandono de las tropas procurando formar nuevas tropas familiares al iniciarse la temporada reproductiva. Las tropas de machos o solteros están compuestas por animales no territoriales de entre uno y cuatro años de edad con un tamaño promedio de 10 individuos (Puig \& Videla 1995), y no existe un liderazgo marcado o relaciones de dependencia entre sus integrantes (Larrieu et al. 1985).

Un aspecto importante dentro de la población de guancos del PNBFJ es el desconocimiento del grado de aislamiento en el que se encuentran estos individuos, por lo que surge la preocupación que implica la depresión por endogamia (Jiménez et al. 1994, De la Torre 2008), que traería como consecuencia la extinción local de este grupo de guanacos en el tiempo, aun cuando la población se incrementó. En todo caso, es necesario encontrar algún tipo de conectividad entre los individuos que usan el PNBFJ e individuos que habiten más allá de los límites del Parque como las poblaciones de Choros $160 \mathrm{~km}$ al norte o las de Illapel $120 \mathrm{~km}$ al sureste del Parque, para que la variación genética se incremente por recombinación durante la reproducción sexual, produciendo nuevas combinaciones de genes y flujo génico por la entrada de nuevos genes desde otras poblaciones (Cabreo \& Camacho 2002).

Uso y selección de cobertura vegetal.- La alimentación, actividad frecuente en los guanacos (Aprile \& Schneider 2009), fue el principal uso que dieron a la cobertura vegetal dentro de sus territorios en el PNBFJ, convirtiendo a esta actividad en la que probablemente guíe la selección. La cobertura vegetal de Matorral Desértico Mediterráneo Interior de Heliotropium stenophyllum y Fluorensia thurifera, presenta los elementos más importantes de la dieta del guanaco (Adesmia sp., Bridgesia sp., Calandrinia sp., Fluorensia sp., Puya sp., Stipa sp., Festuca sp., Bahia sp.). Aunque se desconoce la frecuencia con la que los machos vigilan a sus tropas, en el PNBFJ esta actividad fue la menos realizada y podría explicarse por la baja presencia de depredadores, con- sidérese que en el Parque se conoce que habitan dos especies de zorros y el puma que aparece ocasionalmente (Jaksic et al. 2004).

En el Parque Nacional Torres del Paine (PNTP), el guanaco seleccionó durante todo el año zonas con poca cobertura vegetal (alto porcentaje de suelo desnudo) y con cobertura de matorral xerófilo, incorporando en verano el uso de las vegas (Iranzo 2011), tipos de cobertura que no se corresponden con los del PNBFJ, pero, coincide en cuanto a formas de plantas adaptadas al déficit hídrico (Iranzo 2011). En general, dentro del PNBFJ el guanaco seleccionó zonas abiertas con vegetación de bajo porte donde se relacionó la disponibilidad de alimento y una buena visibilidad, coincidiendo con los resultados de Sarno et al. (2008) y Pedrana et al. (2010). Se asume que una especie selecciona los recursos que mejor puedan satisfacer sus necesidades de vida, y que los recursos de alta calidad serán seleccionados por encima de los de baja calidad (Manly et al. 2002), por lo tanto, el Matorral Desértico Mediterráneo Interior de H. stenophyllum y $F$. thurifera estarían presentando los recursos alimenticios de más alta calidad para el guanaco.

Finalmente, monitorear los individuos reintroducidos constituye una actividad relevante, ya que la respuesta que la población manifieste ante las nuevas condiciones de hábitat, constituye un paso fundamental para inferir ajustes en su manejo e incluso, permitirá establecer su rol ecológico en los sistemas naturales (Estes 1996, Seddon 1998). Marcar los guanacos con collares satelitales para hacer un seguimiento de los movimientos de las tropas y establecer los territorios de cada tropa, permitirá realizar un mejor monitoreo y medición el tamaño poblacional, como ya se realizó en la precordillera de los Andes de la provincia de Choapa en la Región de Coquimbo (González et al. 2008). Además, esta herramienta permitirá conocer si existe una tropa permanente dentro del Parque o todas tienen al Parque como parte de sus territorios, así como determinar si los guanacos del Parque funcionan como fuente y establecer cuáles son los sumideros que reciben a los nuevos individuos que son expulsados de sus tropas. También es altamente recomendable incrementar los datos sobre uso de tipos de cobertura vegetal que realiza el guanaco dentro del Parque, incluso disminuir la escala. Este estudio mostró la selección del Matorral Desértico Mediterráneo Interior de H. stenophyllum y F thurifera, sin embargo, es necesario incrementar este conocimiento para el manejo y conservación de la especie tanto dentro como fuera del Parque, es decir en la zona de amortiguamiento que es donde estaría parte de los territorios de algunas tropas, incluso comprobar el impacto de la competencia por alimento con otros herbívoros como el ganado vacuno, equino o caprino del entorno del parque, como se realizó en el PNTP (Iranzo 2011).

\section{Literatura citada}

Aprile G. \& O. Schneider. 2009. Respuesta comportamental de guanacos (Lama guanicoe) translocados y reintroducidos en el Parque Nacional Quebrada del Condorito, Provincia de Córdoba, Argentina. APRONA Boletín Científico. 41: 69-87. 
Armstrong P. 2007. Using adaptative management to determine requirements of reintroduced populations: the case of the New Zealand hihi. Journal of Applied Ecology 44:953-962. https://doi.org/10.1111/j.13652664.2007.01320.x

Baldi R., A. Pelliza-Sbriller, D. Elston \& S.D. Albon. 2004. High potential for competition between guanacos and sheep in Patagonia. Journal of Wildlife Management 68(4):924-938. https://doi.org/10.2193/0022541X(2004)068[0924:HPFCBG]2.0.CO;2

Baldi R., D. de Lamo, M. Failla, P. Ferrando, M. Funes, P. Nugent, S. Puig, S. Rivera \& J. von Thüngen. 2006. Plan Nacional de Manejo del Guanaco (Lama guanicoe) - República Argentina - Secretaria de Ambiente y Desarrollo Sustentable de la Nación. 37pp.

Barri F. \& M. Cufré. 2014. Supervivencia de guanacos (Lama guanicoe) reintroducidos con y sin período de preadapatación en el Parque Nacional Quebrada del Condorito, Córdoba, Argentina. Mastozoología Neotropical, 21(1):9-16.

Barri F. \& M. Fernández. 2011. Foraging and vigilance time allocation in a guanaco (Lama guanicoe) population reintroduced in Quebrada del Condorito National Park (Córdoba, Argentina). Acta Ethologica 14:103-107. https://doi.org/10.1007/s10211-011-0098-5

Bonino N. \& E. Fernández. 1994. Distribución general y abundancia relativa de guanacos (Lama guanicoe) en diferentes ambientes de Tierra del Fuego, Argentina. Ecología Austral: 4:79-85.

Cabrero J. \& J. Camacho. 2002. Fundamento de Genética de Poblaciones. En M. Soler (Ed), Evolución. La Base de la Biología (pp. 83-126). España. Proyecto Sur Ediciones.

Cajal J. L. 1983. Censo de camélidos y comportamiento del guanaco en la Reserva de San Guillermo (San Juan). Subsecretaría de Ciencia y Tecnología, Buenos Aires, Argentina. 33 pp.

Cook V. 2012. Lineamientos para una propuesta de programa de reintroducción de guanacos Lama guanicoe en la zona núcleo del Parque Nacional Bosque Fray Jorge. Memoria para optar el título profesional de Ingeniero en gestión y manejo de áreas silvestres protegidas. Instituto Profesional IPLACEX. Escuela de Negocios. Santiago, Chile. 46 pp.

Cook V., C. Chávez-Villavicencio \& E. Tabilo. 2013. Proyecto: Programa de monitoreo de largo plazo para la población de guanacos (Lama guanicoe) en el Parque Nacional Bosque Fray Jorge. GesNat Consultores y Corporación Nacional Forestal. Coquimbo, Chile. 47 pp.

Cook V. 2016. Guanaco (Lama guanicoe), objeto de conservación prioritario para el Parque Nacional Bosque Fray Jorge, Región de Coquimbo. Biodiversidata 4: 78-85.

De La Torre J. 2008. Genética de la Conservación. Departamento de Biología. Facultad de Ciencias. Universidad Autónoma de Madrid. Página de Docencia. URL: http:// www.uam.es/personal_pdi/ciencias/joaquina/ Acceso: $30 / 1 / 2014$.

Estes J. 1996. Predators and ecosystem management. Wildlife Society Bulletin 24: 390- 396.

Gader R. 1982. Informe sobre el relevamiento aéreo de guanacos en el Dpto. Collon-Curá. Dirección de Ecología Animal, Neuquén - Argentina. 4 pp.
González B., F. Novoa \& K. Saffer. 2008. Desplazamiento altitudinal y rango de hogar de guanacos (Lama guanicoe) mediante seguimiento con collares satelitales. Biodiversidad de Fauna en Minera Los Pelambres. Ediciones del Centro de Ecología Aplicada Ltda. 57 p.

Franklin W. L. 1983. Contrasting socioecologies of South America's wild camelids: The vicuña and the guanaco. Pp. 573-629, en: Advances in the Study of Mammalian Behavior (JF Eisenberg y DG Kleiman, eds.). Special Publication 7. The American Society of Mammologists. Shippeusburg, Pennsylvania.

Fundación DeSdelChaco (Fundación Desarrollo Sustentable Del Chaco). 2004. Monitoreo de guanacos, Lama guanicoe en el Chaco Paraguayo. Asunción - Paraguay. 30 pp.

Grimberg M. (ed). 2010. Plan Nacional de Conservación del Guanaco (Lama guanicoe Müller, 1776) en Chile 2010 - 2015. Macrozona Norte y Centro. Menssage, Chile. 42 pp.

IUCN (Unión Mundial para la Conservación). 2013. Guidelines for Reintroductions and Other Conservation Translocations. Version 1.0. Gland, Switzerland: IUCN Species Survival Commission, viiii $+57 \mathrm{pp}$.

Jaksic F., E. Silva-Aranguiz \& S. Silva. 2004. Fauna del Parque Nacional Bosque Fray Jorge: una re visión bibliográfica. En: F.A. Squeo, J.R. Gutiérrez \& I.R. Hernández (eds). Historia Natural del Parque Nacional Bosque Fray Jorge: 93-114. Ediciones Universidad de La Serena, La Serena, $307 \mathrm{pp}$.

Jiménez J.C.A., K.C.A. Hughes, G. Alaks, L. Graham \& R.C. Lacy. 1994. An experimental study of inbreeding depression in a natural habitat. Science, 266: 271-273. https:// doi.org/10.1126/science.7939661

Johnson D. 1999. The insignificance of statistical significance testing. Journal of Wildlife Management, 63:763-772. https://doi.org/10.2307/3802789

Larrieu E.J., R.O. Bigatti \& N.R. Oporto. 1985. La Reproducción de los Camélidos Sudamericanos en la Argentina. En: J.L Cajal \& J.N. Amaya (Eds.). Estado Actual de las Investigaciones sobre Camélidos en la República Argentina. SECyT, Buenos Aires, Argentina. 101 - 109 pp.

Luebert F. \& P. Pliscoff. 2009. Sinopsis bioclimática y vegetacional de Chile. Cobertura actualizada. Escala 1:250.000, cobertura nacional. Sistema de Información Ambiental Geográfica, Ministerio del Medio Ambiente, Gobierno de Chile.

Manly B.F.., L.L. Mcdonald, D.L. Thomas, T.L. Mcdonald \& W.P. Erickson. 2003. Resource selection by animals: Statistical design and Analysis for field studies. Second edition. London: Kluwer Academic Publishers.

Marín J. 2008. Guanaco. Ficha de antecedentes de la especie. URL: www.conama.cl. Acceso: 20/1/2016.

Marquet P.A., F. Bozinovic, G.A. Bradshaw, C. Cornellius, H. González, J.R. Gutiérrez, E. Hajek, J.C.A. Lagos, F.J. LópezCortes, L. Núñez, E.F. Rosello, C. Santoro, H. Samaniego, V.G. Standen, J.C. Torres-Mura \& F.M. Jaksic. 1998. Ecosistemas del desierto de Atacama y área Andina adyacente. Revista Chilena de Historia Natural 71:593-617.

Novoa J.E. \& D.A. López. 2001. IV Región: El Escenario Geográfico Físico. En: F.A. Squeo, G. Arancio \& J.R. Gutiérrez (eds) Libro Rojo de la Flora Nativa y de los Sitios Prioritarios para su Conservación: Región de Coquimbo: 13-28. Ediciones Universidad de La Serena, La Serena, $372 \mathrm{pp}$. 
Paskoff R. 1993. Geomorfología de Chile Semiárido. Ediciones Universidad de La Serena. 321 pp.

Pedrana J., J. Bustamante, A. Travaini \& A. Rodríguez. 2010. Factors influencing guanaco distribution in southern Argentine Patagonia and implications for its sustainable use. Biodiversity and Conservation, 19: 3499-3514. https://doi.org/10.1007/s10531-010-9910-1

Pouget M., E. Caviedes, P.H. Hamelin, D. Remy, R. Mathieu, V. Lira \& D. Álvarez. 1996. Ambiente árido y desarrollo sustentable: la provincia de Limarí. Coedición ORSTOMCEZA, Santiago, 103 pp.

Puig S. \& F. Videla. 1995. Comportamiento y Organización Social del Guanaco. pp 97 - 118. En: S. Puig (ed.) Técnicas para el manejo del guanaco. IUCN, Gland, Suiza.

Puig S., F. Videla, M.I. Cona \& S.A. Monge. 2001. Use of food availability by guanacos (Lama guanicoe) and livestock in Northern Patagonia (Mendoza, Argentina). Journal of Arid Environments, 47(3):291-308. https://doi. org/10.1006/jare.2000.0677

Raedeke K.J. 1978. El guanaco de Magallanes, Chile. Su distribución y biología. Chile, Corporación Nacional Forestal. Publicación Técnica $\mathrm{N}^{\circ} 1.200 \mathrm{pp}$.

Raedeke K.J. 1979. Population Dynamics and Socioecology of the Guanaco (Lama Guanicoe) of Magallanes. Dissertation for Doctor of Philosophy. University of Washington. $404 \mathrm{pp}$.

R Development Core Team. 2018. R: A language and environment for statistical computing. R Foundation for Statistical Computing, Vienna, Austria. ISBN 3-90005107-0. URL: http://www.R-project.org

Rundel P. W. \& M. Mahu. 1976. Community structure and diversity of a coastal fog zone in northern Chile. Flora 165: 493-505. https://doi.org/10.1016/S03672530(17)31888-1

Sarasqueta D. 2001. Cría y Reproducción de Guanacos en Cautividad Lama guanicoe. Comunicación Técnica $\mathrm{N}^{\circ} 110$ Recursos Naturales Fauna. Bariloche - Argentina. 61 pp.

Sarno R.J., M.S. Bank, H.S. Stern \& W.L. Franklin. 2003. Forced dispersal of juvenile guanacos (Lama guanicoe): causes, variation, and fates of individuals dispersing at different times. Behaviour Ecology Sociobiology 54:2229. https://doi.org/10.1007/s00265-003-0604-5
Sarno R. J., M.M. Grigione \& L.D. Arvidson. 2008. Lack of response of an open-habitat ungulate to the presence of predator urine. Revista Chilena de Historia Natural, 81: 179-183. https://doi.org/10.4067/S0716078X2008000200003

Sarria Castro M. \& L.C. Silva Ayçaguer. 2004. Las pruebas de significación estadística en tres revistas biomédicas: una revisión crítica. Revista Panamericana de Salud Pública 15(5):300-6. https://doi.org/10.1590/S102049892004000500003

Schamberger M. \& G. Fulk. 1974. Mamíferos del Parque Nacional Fray Jorge. IDESIA 3:167-179.

Seddon P. 1998. Improving the rigor of re-introduction project assessment, planning and execution: report on the round table discussion on bird-introductions. Reintroduction News. Newsletter of the Re-introduction Specialist Group. Species Survival Commission (IUCN). 16: 3-5. Nairobi, Kenya.

Seddon P. 2007. Combining the field of reintroduction biology and restoration ecology. Conservation Biology 21:1387-1390.

Seddon P. \& P.S. Soorae. 1999. Guidelines for subspecies substitutions in wildlife restoration projects. Conserva 7 tion Biology 13:177-181. https://doi.org/10.1046/j.15231739.1999.97414.x

Soorae P. S. 2011. IUCN Global re-introduction perspec $\neg$ tives: 2011. More case studies from around the globe. Gland, Switzerland and Cambridge.

Squeo F.A., D. López \& J.E. Novoa. 2001. Listado y antecedentes de los sitios prioritarios para la conservación de la flora nativa con problemas de conservación de la Región de Coquimbo. En: F.A. Squeo, G. Arancio \& J.R. Gutiérrez (eds). Libro Rojo de la Flora de la Región de Coquimbo y de los Sitios Prioritarios para su Conservación: Región de Coquimbo: 195-202. Ediciones Universidad de La Serena, La Serena, $371 \mathrm{pp}$.

Agradecimientos:

A los Guardaparques del PNBFJ y voluntarios que ayudaron en los conteos. A Fernando Barri por facilitar bibliografía. A los revisores anónimos que con sus aportes mejoraron este artículo.

Conflicto de intereses:

Los autores no incurren en conflictos de intereses.

Rol de los autores:

VC-M, CC-V, PM-P: Realizaron el diseño experimental, analizaron los datos, redactaron y aprobaron el manuscrito; ET-V: analizó los datos, redactó y aprobó el manuscrito.

Fuentes de financiamiento:

Corporación Nacional Forestal de Chile.

Aspectos éticos / legales:

Este trabajo no involucró colecta o preservación de especímenes y no usó técnicas invasivas para su desarrollo. Este trabajo no incurrió en ningún problema legal. El sacrificio de animales en 1994 se realizó bajo los estándares del Servicio Agrícola Ganadero del Ministerio de Agricultura de Chile y en salvaguarda de los individuos liberados y el público visitante. 